\title{
An Image Enhancement Framework for Fault Diagnosis and Feature Extraction
}

\author{
Malkit Singh ${ }^{1}$, Gurjinder Singh ${ }^{2}$ \\ ${ }^{1,2}$ Guru Gobind Singh College of Science and Engineering Guru Kashi University Talwandi Sabo (Bathinda), India
}

\begin{abstract}
Image enhancement refers to accentuation, or sharpening, of image features such as edges, boundaries, or contrast to make a graphic display more useful for display and analysis. The enhancement process does not increase the inherent information content in the data. But it does increase the dynamic range of the chosen features so that they can be detected easily. The greatest difficulty in image enhancement is quantifying the criterion for enhancement. Therefore, a large number of image enhancement techniques are empirical and require interactive procedures to obtain satisfactory results. Many image enhancement techniques are based on spatial operations performed on local neighborhoods of input pixels. Often, the image is convolved with a finite impulse response filter called spatial mask. This study will highlight various image enhancement techniques along with their benchmark results. Image enhancement technologies have attracted much attention during the diagnosis process. The principle objective of image enhancement techniques is to process an input image so that the resultant image is more suitable than the original image for specific application. Traditional global histogram equalization usually causes excessive contrast. Enhancement while local histogram equalization may cause block effect. To overcome these problems, a new method for image contrast enhancement is developed. The novelty of the proposed method is that the weighted average of the histogram equalized, gamma corrected and the original image are combined to obtained the enhanced processed image. The proposed algorithm not only achieve contrast enhancement but also preserves the brightness level. Experimental results show that the proposed algorithm has good performance on enhancing contrast and visibility for a majority of images.
\end{abstract}

Keywords: Histogram Equalization, Image Enhancement, Image Filtering, PSNR, MSE, NCC and NAE

\section{Introduction}

Image enhancement refers to accentuation, or sharpening, of image features such as edges, boundaries, or contrast to make a graphic display more useful for display and analysis. The enhancement process does not increase the inherent information content in the data. But it does increase the dynamic range of the chosen features so that they can be detected easily. The greatest difficulty in image enhancement is quantifying the criterion for enhancement. Therefore, a large number of image enhancement techniques are empirical and require interactive procedures to obtain satisfactory results. Many image enhancement techniques are based on spatial operations performed on local neighborhoods of input pixels. Often, the image is convolved with a finite impulse response filter called spatial mask. The spatial domain methods are deals directly with pixel values composing an image [1]. There are different types of spatial operations that can be applied in the spatial domain for image enhancement: Noise smoothing, Median filtering, Low-pass, band-pass and high-pass filtering. Also, there are two different types of spatial filtering techniques: spatial linear enhancement techniques, and spatial non-linear enhancement techniques [2].

\subsection{Image enhancement techniques}

The choice of enhancement technique has a direct impact on the final result, since the image quality greatly influences the subsequent analysis. However in the literature, relatively little attention has been given to this pre-processing step. In the following, we briefly discuss the ten enhancement techniques that we evaluated in our study, for further details we refer to the original papers.

\subsubsection{Median filter}

A commonly employed noise removal technique, also known as order-statistic filter, which replaces the value of a pixel by the median of the intensity values in the neighborhood of that pixel.

\subsubsection{Gaussian filter}

A smoothing filter, defined by a Gaussian kernel, which shows lower blurring effects compared to simpler averaging filters.

\subsection{3 $\alpha$-trimmed filter}

A non-linear window-based filter. It can be considered as a hybrid filter derived from mean and median filters. If the pixel values $X_{k}$ within a window around location $(i, j)$ are sorted in ascending order $\mathrm{x} 1 \leq \mathrm{x} 2 \leq \cdots \leq \mathrm{xN}$, then the output of the $\alpha$-trimmed filters defined as

$\mathrm{x}_{\mathrm{ij}}=1 / \mathrm{N}(1-\alpha 1-\alpha 2)$ Summation $(\mathrm{x} \mathrm{k}), 0 \leq \alpha 1, \alpha 2<0.5$,

................ (1)

where $\alpha 1$ and $\alpha 2$ are the parameters of the filter.

\subsubsection{Band-pass $\varepsilon$ filter:}

A non-linear filter which reduces image noise while preserving edge information. A difference of Gaussian (DoG) approach is employed leading to

$\mathrm{y}(\mathrm{k}, \mathrm{l})=\Phi \varepsilon 1, \mathrm{M} 1[\mathrm{x}(\mathrm{k}, \mathrm{l})]-\gamma \Phi \varepsilon 2, \mathrm{M} 2[\mathrm{x}(\mathrm{k}, \mathrm{l})]$

with $\Phi \varepsilon, \mathrm{M}[\mathrm{x}(\mathrm{k}, \mathrm{l})]=\mathrm{x}(\mathrm{k}, \mathrm{l})+$

$$
\text { Summation }(a(i, j) F(x(k+i, 1+j)-x(k, 1))
$$

where $x(k, 1)$ is the 2-dimensional input signal, $F(x)=x$ for $(-\varepsilon \leq \mathrm{x} \leq \varepsilon)$ and 0 otherwise, and $\gamma$ is a filter coefficient.

\subsubsection{Anisotropic diffusion filtering}

An iterative algorithm which smoothens an image using a diffusion function. Smoothing is formulated as a diffusive process which is stopped at boundaries by selecting locally adaptive diffusion strengths. Diffusion functions allow the diffusion process to take place in the interior of regions 


\section{International Journal of Science and Research (IJSR) \\ ISSN (Online): 2319-7064}

Index Copernicus Value (2015): 78.96 | Impact Factor (2015): 6.391

while not affecting edges. The anisotropic diffusion equations defined as

$$
\text { It }=\operatorname{div}(\mathrm{c}(-\mathrm{x}, \mathrm{t}) \nabla \mathrm{u}(-\mathrm{x}, \mathrm{t})) \text {, }
$$

where diffusion is controlled by varying $\mathrm{c}(-\mathrm{x}, \mathrm{t})$, and $\mathrm{u}(-\mathrm{x}, \mathrm{t})$ represents the image intensity. The resulting image is obtained after optimizing $\mathrm{c}(-\mathrm{x}, \mathrm{t})$.

\subsubsection{Bilateral filter}

A non-iterative, relatively simple algorithm which smoothens an image while preserving edges by means of a non-linear combination of near by image values based on both their spatial closeness $\mathrm{c}(\xi$, $\mathrm{x})$ and their photometric similarity $\mathrm{s}(\mathrm{f}(\xi), \mathrm{f}(\mathrm{x}))$. It is suggested that the two pixels can be close to one another, that is occupy nearby spatial location $(\mathrm{c}(\xi, \mathrm{x}))$ or they can be similar to one another, that is, have nearby values $(\mathrm{s}(\mathrm{f}(\xi), \mathrm{f}(\mathrm{x})))$. Bilateral filtering, basically the combination of domain and range filtering, is defined as

$\mathrm{h}(\mathrm{x})=$ Integral $(\Omega(\mathrm{x}) \mathrm{f}(\xi) \mathrm{c}(\xi, \mathrm{x}) \mathrm{s}(\mathrm{f}(\xi), \mathrm{f}(\mathrm{x}))) \mathrm{d} \xi /$

Integral $(\Omega(\mathrm{x}) \mathrm{c}(\xi, \mathrm{x}) \mathrm{s}(\mathrm{f}(\xi), \mathrm{f}(\mathrm{x}))) \mathrm{d} \xi$

\subsubsection{Bilateral enhancer}

Extends the concept of bilateral filters so that edge preserving smoothing and selective sharpening is performed simultaneously. A weighted average is utilized that is independent of the design of $c($.$) and s($.$) in Eq. (5).$ Furthermore, it considers a special case when $\xi=\mathrm{x}$ and adds a constant $\mathrm{g}$ in this case. This does not change the nature of the filter, but for $\xi=\mathrm{x}$, the contribution of $\mathrm{c}($.$) and \mathrm{s}($.$) is$ summarized into $g$. The bilateral enhancer is defined as $\mathrm{j}(\mathrm{x})=\mathrm{g} \mathrm{f}(\mathrm{x})+$ Integral $(\mathrm{c}(\xi, \mathrm{x}) \mathrm{p}(\mathrm{f}(\mathrm{x}), \mathrm{f}(\xi))) \mathrm{d} \xi$

$$
\text { where } g=c(x, x) s(f(x), f(x)) \text {. }
$$

\subsubsection{Wavelet filtering}

Uses an adaptive data-driven threshold for image de-Noising employing wavelet soft-Thresholding .The method we employ is also referred to as Bayes-Shrink which performs soft-thresholding, with a data-driven, sub-band dependent threshold derived in a Bayesian framework.

\subsubsection{Non local means (NLM):}

A non-iterative algorithm which smoothens an image by a non-local averaging of all pixels in the image. The weights depend on the similarity between two pixels which are defined by the similarity of the intensity grey level in a square neighborhood of fixed size. The similarity is measured as a decreasing function of weighted Euclidean distance. The NLM filter is defined as

NLM[v](i) =Summation $(w(i, j) v(j))$ where the weights $w(i, j)$ depend on the similarity between pixels $\mathrm{i}$ and $\mathrm{j}$.

\subsubsection{Adaptive damped wave equation (ADWE)}

A stable approximated version of an enhancement operator proposed using Forward-and-Backward (FAB) TelegraphDiffusion (Ted) equations. The Ted equation of damped wave is used in the context of image processing for its characteristic behavior of smoothing. The basic Ted equation is

$$
\mathrm{u}_{\mathrm{u}}-\nabla \cdot((\mathrm{k}(|\nabla \mathrm{u}|)) \nabla \mathrm{u})+\mathrm{cu}_{\mathrm{t}}=0
$$

where $u(t=0)$ is the input image and $\mathrm{c}$ is a positive damping constant. The elasticity coefficient $\mathrm{k}$ controls the degree of smoothing. Furthermore, a backward component $\mu$ is introduced to allow $\mathrm{k}$ to drop below 0 in order to minimize the effect of blurring near edges.

\section{Related work to proposed image enhancement techniques}

\subsection{Image Enhancement with Log Transformation}

The general form of the $\log$ transformation shown in equation below

$$
\mathrm{S}=\mathrm{c} \log (1+\mathrm{r})
$$

where $c$ is a constant, and it is assumed that $r>=0$. The shape of the log curve describes that this transformation maps a narrow range of low gray-level values in the input image into a wider range of output levels. The opposite is true of higher values of input levels. We would use a transformation of this type to expand the values of dark pixels in an image while compressing the higher-level values. The opposite is true of the inverse log transformation. Any curve having the general shape of the log functions would accomplish this spreading/compressing of gray levels in an image. In fact, the power-law transformations discussed in the next section are much more versatile for this purpose than the log transformation. However, the log function has the important characteristic that it compresses the dynamic range of images with large variations in pixel values. A classic illustration of an application in which pixel values have a large dynamic range is the Fourier spectrum. At the moment, the main concern is only with theimage characteristics of spectra. It is not unusual to encounter spectrum values

\subsection{Image Enhancement By Power Law Transformations}

Power-law transformations have the basic form

$$
\mathrm{S}=\mathrm{C} * \mathrm{R}^{\mathrm{g}}
$$

where $c$ and $g$ are positive constants. Sometimes this equation is written as to account for an offset (that is, a measurable output when the input is zero). However, offsets typically are an issue of display calibration and as a result they are normally ignored in above equation. Plots of $s$ versus $r$ for various values of $g$ are shown in Fig. 1.1. As in the case of the log transformation power-law curves with fractional values of $g$ map a narrow range of dark input values into a wider range of output values, with the opposite being true for higher values of input levels. Unlike the log function, however, we notice here a family of possible transformation curves obtained simply by varying g. As expected, we see in Fig. 1.1 that curves generated with values of $g>1$ have exactly the opposite effect as those generated with values of $g<1$. Finally, we note that above equation reduces to the identity transformation when $\mathrm{c}=\mathrm{g}=1$.A variety of devices used for image capture, printing, and display respond according to a power law. [3, 4] By convention, the exponent in the power-law equation is referred to as gamma (as $\mathrm{g}$ ). The process used to correct this power law response phenomenon is called gamma correction. For example, cathode ray tube (CRT) devices have an intensity-to-voltage response that is a power function, with exponents varying from approximately 1.8 to 2.5. With reference to the curve for $g=2.5$ in Fig. 1.1, wesee that such display systems would tend to produce images that are darker than intended.This effect is illustrated in Fig. 1.1 shows a simple gray-scale linear wedge input into a CRT

\section{Volume 6 Issue 1, January 2017}




\section{International Journal of Science and Research (IJSR) \\ ISSN (Online): 2319-7064}

Index Copernicus Value (2015): 78.96 | Impact Factor (2015): 6.391

monitor. As expected, the output of the monitor appears darker than the input. Gamma correction in this case is straightforward.
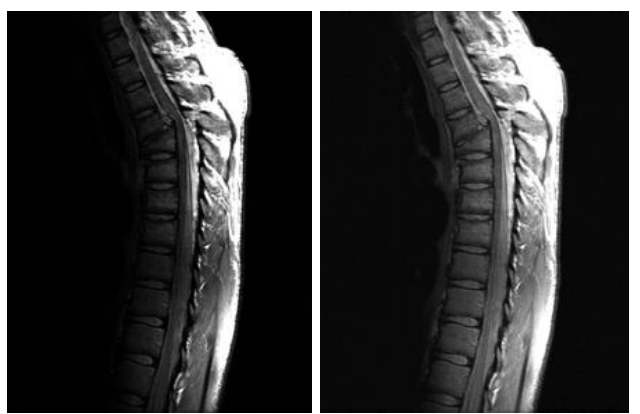

Figure 1: Magnetic Resonance image of fractured human spine (Left image is original image and right image is enhanced by power law with $\mathrm{g}=0.6$ )

\subsection{Image Enhancement By Histogram Equalization}

Consider for a moment continuous functions, and let the variable $r$ represent the gray levels of the image to be enhanced. In the initial part of our discussion we assume that $r$ has been normalized to the interval $[0,1]$, with $\mathrm{r}=0$ representing black and $\mathrm{r}=1$ representing white. Later, we consider a discrete formulation and allow pixel values to be in the interval $[0, \mathrm{~L}-1]$.For any $r$ satisfying the aforementioned conditions, we focus attention on transformations of the form

$$
\mathrm{s}=\mathrm{T}(\mathrm{r}) 0<\mathrm{r}<1
$$

that produce a level $s$ for every pixel value $r$ in the original image. For reasons that will become obvious shortly, we assume that the transformation function $\mathrm{T}(\mathrm{r})$ satisfies the following conditions:

(a) $\mathrm{T}(\mathrm{r})$ is single-valued and monotonically increasing in the interval

$0<\mathrm{r}<1$; and

(b) $0<\mathrm{T}$ (r) $<1$ for all $0<\mathrm{r}<1$.

The requirement in (a) that $\mathrm{T}(\mathrm{r})$ be single valued is needed to guarantee that the inverse transformation will exist, and the monotonicity condition preserves the increasing order from black to white in the output image. A transformation function that is not monotonically increasing could result in at least a section of the intensity range being inverted, thus producing some inverted gray levels in the output image. While this may be a desirable effect in some cases, that is not what we are after in the present discussion .Finally, condition (b) guarantees that the output of gray levels is the same range as the input levels. Figure 1.2 gives an example of a transformation function that satisfies these two conditions. The inverse transformation from $s$ back to $r$ is denoted in above equation. It can be shown by example that even if $\mathrm{T}(\mathrm{r})$ satisfies conditions(a) and (b), it is possible that the corresponding inverse may fail to be single valued.

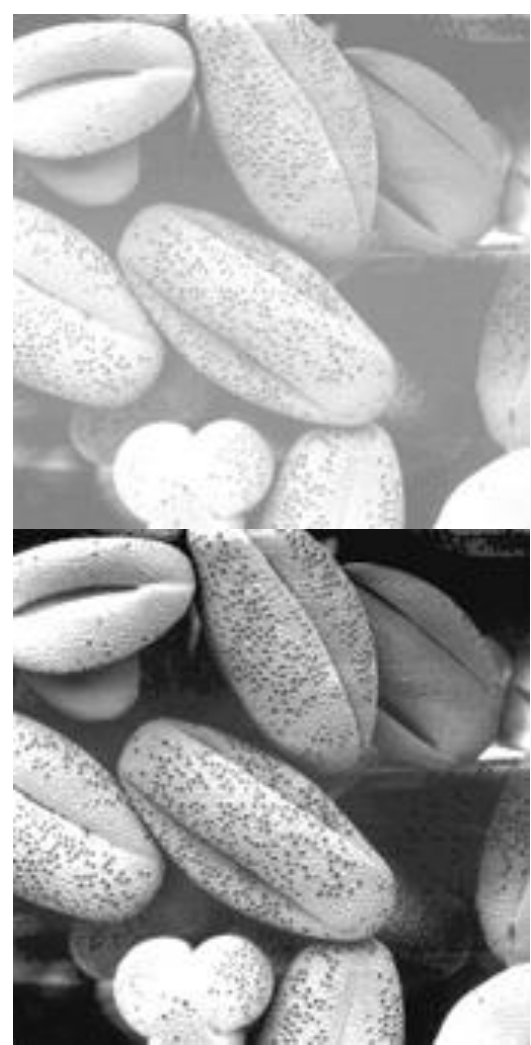

Figure 2: Wheat grains image taken in Australian University Canberra (Left one is originally captured and right one is enhanced by histogram equalization)

\section{Proposed Algorithm for Image Enhancement}

There are several methods are adopted for contrast enhancement of remote sensing images. Most of them are modifications of Histogram Equalization (HE) [5]. In brightness preserving Bi-Histogram Equalization (BBHE) [6] two separate histograms are taken from the same image and then equalized separately, where the first one is the histogram of intensities that are less than the mean intensity and the second one is the histogram of intensities that are greater than the mean intensity. In Dualistic Sub-image Histogram Equalization (DSIHE) [7] two separate histograms are created based on the median instead of mean. The Recursive Mean Separation Histogram Equalization (RMSHE) [4] enhances image by iterating BBHE technique. Recently, the gain controllable clipped histogram equalization (GC-CHE) [9] method performs clipped histogram equalization for preserving the brightness of an image. In Demerol's method of contrast enhancement the singular value decomposition matrix [10][11] was applied to the low level sub band. This method distorting the image details at low and high intensity regions.

\section{Proposed Algorithm}

Step 1: Read the input image

Step 2: Convert RGB to Gray for that image

Step 3: Find out DCT and Inverse DCT

Step 4: Merge with original image the image obtained in step 3.

Step 5: Extract histogram of the image taken in step1.

Step 6: Run histogram equalization algorithm

Step 7: Apply fusion of image taken from step4 and step6. 


\section{International Journal of Science and Research (IJSR) \\ ISSN (Online): 2319-7064}

Index Copernicus Value (2015): 78.96 | Impact Factor (2015): 6.391

Step 8: Compare the performance parameters of this enhanced image with original image.

\section{Simulation Tool Used}

Here in this work, Computer Vision toolbox of Matlab 2013 is used. It includes various features of processing images through standard techniques. These techniques are recently developed by Matlab developers. Computer Vision System Toolbox provides algorithms and tools for the design and simulation of computer vision and video processing systems. The toolbox includes algorithms for feature extraction, motion detection, object detection, object tracking, stereo vision, video processing, and video analysis. Tools include video file I/O, video display, drawing graphics, and compositing. Capabilities are provided as MATLAB functions, MATLAB System objects, and Simulink blocks. For rapid prototyping and embedded system design, the system toolbox supports fixed-point arithmetic and C-code generation. Its Key Features includes; Feature detection, including FAST, Harris, Shi \& Tomasi, SURF, and MSER detectors Feature extraction and putative feature matching, Object detection and tracking, including Viola-Jones detection and CAM Shift tracking, Motion estimation, including block matching, optical flow, and template matching, RANSAC-based estimation of geometric transformations or fundamental matrices, Video processing, video file I/O, video display, graphic overlays, and compositing, Block library for use in Simulink.

\section{Generation of Corner Matrix In MATLAB}

The implementation of the Proposed Image Enhancement algorithm is done by using Computer Vision Toolbox in MATLAB. The implementation was done in 5 phases:-

1. Image Acquisition

2. RGB to Gray Scale Conversion

3. Histogram Extraction of original image

4. Fusion of original image \& Histogram equalized image.

5. Performance measurement of the proposed Algorithm.

The following are some snapshots of the inputs, inference engine and output on the editor:-

\subsection{Implementation of Histogram Equalization Algorithm}

One input vector gives connectivity of regions of Image to Object Particles. Any Randomly selected image will make connectivity of domain with the observed input point. Here in this interface user need to load an image from domain of pre-loaded images. One of them got read while selection from drop down list appeared in the interface.

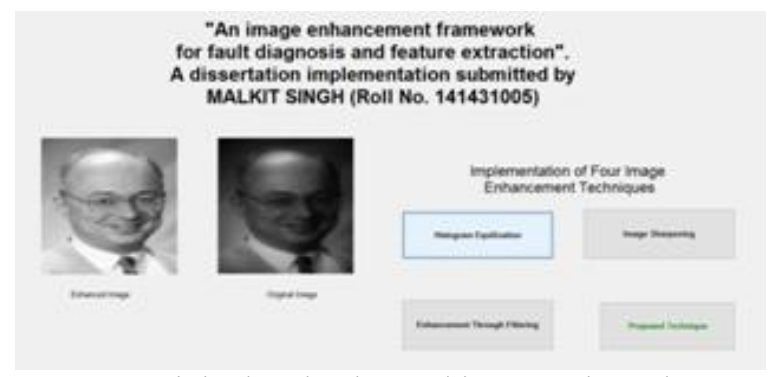

Figure 3: Original and enhanced image using Histogram Equalization Approach

\subsection{Interface Showing Performance Parameters for} Histogram Equalization Image Enhancement Technique

Histogram equalization is a common technique for enhancing the appearance of images. Suppose we have an image which is predominantly dark. Then its histogram would be skewed towards the lower end of the grey scale and all the image detail is compressed into the dark end of the histogram. If we could 'stretch out' the grey levels at the dark end to produce a more uniformly distributed histogram then the image would become much clearer .Loading of an image is done through imread() method in Matlab, source location of image must be determined in order to load it to the Computer Vision database. Image formats like tif, jpeg, bmp.. Etc. are accepted.

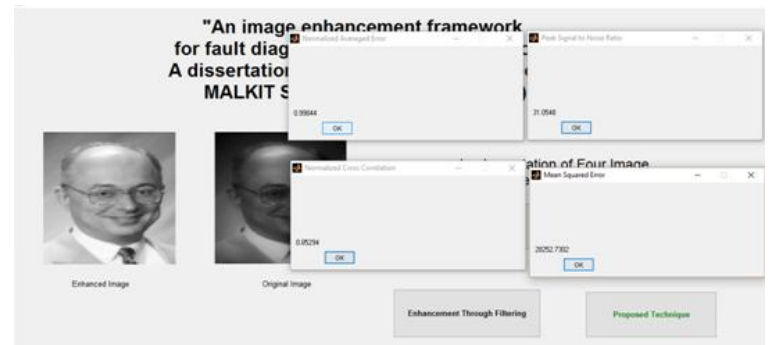

Figure 4: Results of PSNR, MSE, NAE and NCC for Histogram Equalization

\subsection{Interface Showing Results of Image Sharpening Techniques}

Image sharpening techniques makes use of filters for image enhancement process. In this interface image sharpening through contrast stretching is performed on an input image. Their discrete cosine transformations followed by inverse discrete cosine transformation sharpen the image. Then results of performance parameters are normalized with Distance Auto Correlation unit transformations.

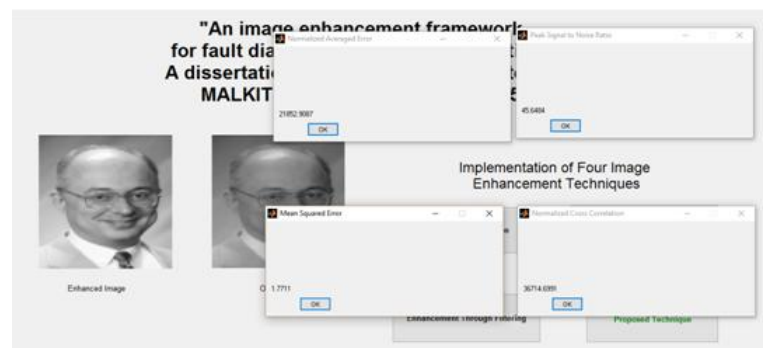

Figure 5: Results of PSNR, MSE, NAE and NCC for Image sharpening using DCT 


\section{International Journal of Science and Research (IJSR) \\ ISSN (Online): 2319-7064}

Index Copernicus Value (2015): 78.96 | Impact Factor (2015): 6.391

5.4 Implementation of Image Enhancement through Filtering

Median filtering is a non-linear, low-pass filtering method, which are used mainly to remove salt-and pepper noise from an image. A median filter can outperform linear low-pass filters on this type of noisy image, because it can potentially removes all the noise without affecting the "clean" pixels. Median filters remove isolated pixels, whether they are bright or dark. Common drawback of the median filtering are destroy small features in the image and computational cost. Computing a two-dimensional median for a $N x N$ window, requires sorting of $N x N$ elements for every image pixel Therefore, using median filtering in any real-time vision system requires a significant computational power. Following figure shows the performance parameters of image enhancement through filtering.

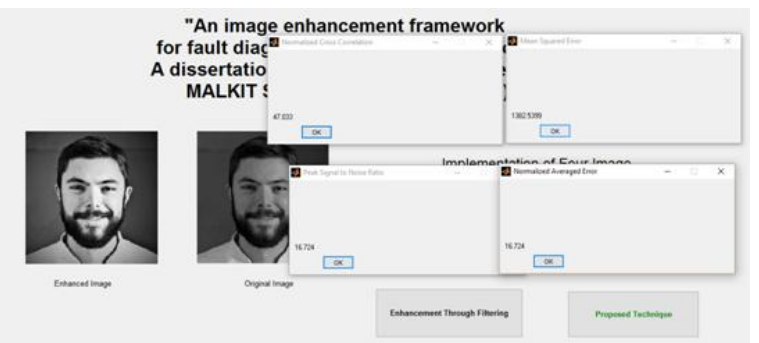

Figure 6: Results of PSNR, MSE, NAE and NCC for Enhancement through Filtering.

\subsection{Enhancement Through Proposed Technique}

The principle objective of image enhancement techniques is to process an input image so that the resultant image is more suitable than the original image for specific application. Traditional global histogram equalization usually causes excessive contrast enhancement while local histogram equalization may cause block effect. To overcome these problems, a new method for image contrast enhancement is developed. The novelty of the proposed method is that the weighted average of the histogram equalized, gamma corrected and the original image are combined to obtained the enhanced processed image .The proposed algorithm not only achieve contrast enhancement but also preserves the brightness level. Experimental results show that the proposed algorithm has good performance on enhancing contrast and visibility for a majority of images. The following figure shows the implementation of proposed image enhancement technique along with its performance parameters.

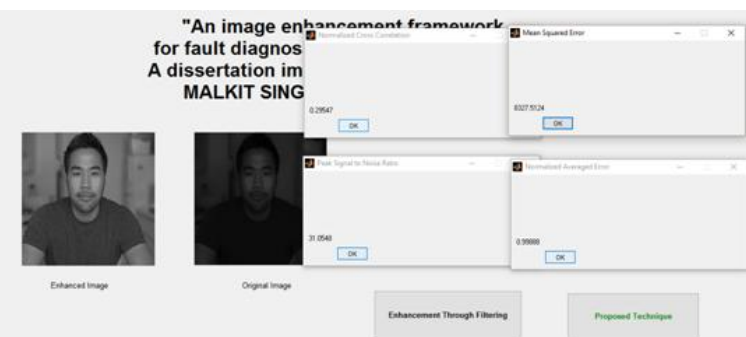

Figure 7: Results of PSNR, MSE, NAE and NCC using Proposed Enhancement Technique.

\section{Outcomes in Terms of Precession and Recall}

Color and texture of image data patterns are observed through sobel-edge detection mechanism. Semantics of similarity between observed image patterns is the matter of distance between images to measure their similarity. This distance is given by Minkowsky and known as Minkowskydistance. It is expected that this distance will provide us similarity measure between images. Sobel-edge detection mechanism will help greatly to correlate two images with their appropriate distance.

$$
\mathrm{L}_{\mathrm{P}}(\mathrm{X}, \mathrm{Y})=\left[\sum\left|\mathrm{x}_{\mathrm{i}}-\mathrm{y}_{\mathrm{i}}\right|^{\mathrm{P}}\right] \frac{1}{P}
$$

Here results of various image retrieval methods are given along with their Minkowsky-distances. HE (Histogram Equalization), IMSH (Image Sharpening), ETF (Enhancement Through Filtering) and PET (Proposed Enhancement Technique).

Table1: Results of Image Retrieval Methods

\begin{tabular}{|c|c|c|c|c|}
\hline & HE & IMSH & ETF & PET \\
\hline Average of precision & 0.72 & 0.74 & 0.9 & 0.78 \\
\hline Average of recall & 0.49 & 0.57 & 0.68 & 0.71 \\
\hline
\end{tabular}

\section{Performance comparison of different Image enhancement techniques}

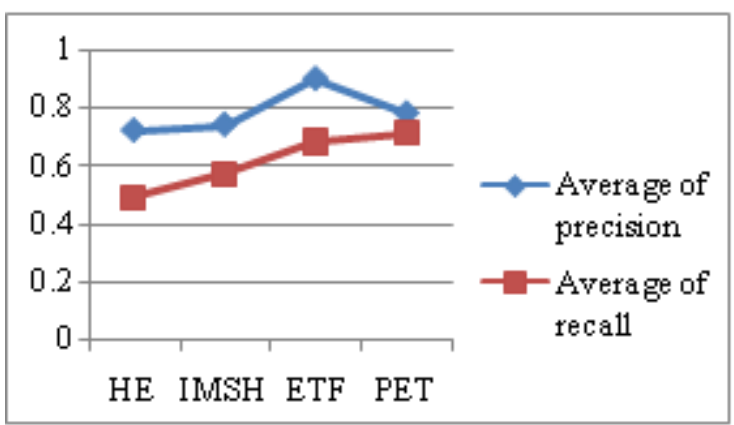

Figure 8: Performance Graph

\section{Conclusion}

The principle objective of image enhancement techniques is to process an input image so that the resultant image is more suitable than the original image for specific application. Traditional global histogram equalization usually causes excessive contrast enhancement while local histogram equalization may cause block effect. To overcome these problems, a new method for image contrast enhancement is developed. The novelty of the proposed method is that the weighted average of the histogram equalized, gamma corrected and the original image are combined to obtained the enhanced processed image. The proposed algorithm not only achieve contrast enhancement but also preserves the brightness level. Experimental results show that the proposed algorithm has good performance on enhancing contrast and visibility for a majority of images

\section{References}

[1] Feng Xiao, Mingquan Zhou, and Guohua Geng, "Detail Enhancement and Noise Reduction with Color Image 
Detection Based on Wavelet Multi-scale," 2011, pp. 1061-1064.

[2] L. Hong, Y. Wan, and A. Jain. "Fingerprint image enhancement: Algorithm and performance evaluation", IEEE Transactions on Pattern Analysis and Machine Intelligence, 20(8):777-789, August 1998.

[3] X. Li, H. Shen, L. Zhang, and H. Li, "Sparse-based reconstruction of missing information in remote sensing images from spectral/temporal complementary information," ISPRS J. Photo gram. Remote Sens.,vol. 106, pp. 1-15, 2015

[4] F. Melgani, "Contextual reconstruction of cloudcontaminated multi temporal multispectral images," IEEE Trans.Geosci. Remote Sens., vol. 44, no. 2, pp. 442-455, Feb. 2006.

[5] Bhabatosh Chanda and Dwijest Dutta Majumder, 2002, Digital Image Processing and Analysis.

[6] R.W.Jr. Weeks, (1996). Fundamental of Electronic Image Processing Bellingham :SPIEP ress

[7] Shih-Chia Huang, Fan-Chieh Cheng, and Yi-Sheng Chiu," Efficient Contrast Enhancement Using Adaptive Gamma Correction With Weighting Distribution", IEEE Transaction on image processing VOL. 22, NO. 3MARCH 2013.

[8] Renjie He, Sheng Luo, Zhanrong Jing, Yangyu Fan "Adjustable Weighting Image Contrast Enhancement Algorithm and Its Implementation", 2011 6th IEEE Conference on Industrial Electronics and Applications

[9] A. Soualhi, K. Medjaher, and N. Zerhouni, "Bearing health monitoring based on Hilbert-Huang transform, support vector machine, and regression," IEEE Trans. Instrum.Meas., vol. 64, no. 1, pp. 52-62, Jan. 2015.

[10] M. Han and J. Pan, "A fault diagnosis method combined with LMD, sample entropy and energy ratio for roller bearings," Measurement, vol. 76, pp. 7-19, Dec. 2015.

[11] N. Foin, J. M. Mari, J. E. Davies, C. Di Mario, and M. J. Girard, "Imaging of coronary artery plaques using contrast-enhanced optical coherence tomography," European Heart Journal-Cardiovascular Imaging, vol. 14, no. 1, pp. 85-85, 2013.

[12]A. Sapena-Bañó, M. Pineda-Sanchez, R. PuchePanadero, J. Martinez-Roman, and D. Mati'c, "Fault diagnosis of rotating electrical machines in transient regime using a single stator current's FFT," IEEE Trans. In strum. Meas., vol. 64, no. 11, pp. 3137-3146, Nov. 2015.

[13] W. Li, A. Monti, and F. Ponci, "Fault detection and classification in medium voltage DC shipboard power systems with wavelets and artificial neural networks," IEEE Trans. In strum. Meas., vol. 63, no. 11,pp. 26512665, Nov. 2014.

\section{Author Profile}

Malkit Singh received BCA in 2012 and Msc (IT) in 2014 Under GNDU(Guru Nanak Dev University, Amritsar).Now His M.Tech(IT) perusing From Guru Kashi University ,Talwandi Sabo (Bathinda),India. 